\title{
Del consultorio de la señora Fagoaga al Hospital Oftalmológico de Nuestra Señora de la Luz
}

\section{From Consultorio de la señora Fagoaga to Hospital Oftalmológico de Nuestra Señora de la Luz}

\author{
Rolando Neri-Vela*
}

Departamento de Historia y Filosofía de la Medicina, Facultad de Medicina, Universidad Nacional Autónoma de México, Ciudad de México, México

\section{Resumen}

En 1750 fue fundado en la ciudad de México un consultorio para atender enfermos de los ojos, que junto con el servicio que había en el Hospital de Pobres dieron asistencia oftalmológica en la capital de la Nueva España. Más tarde se fundó el Instituto Valdivielso, llamado posteriormente Hospital Oftalmológico de Nuestra Señora de la Luz. En este hospital se formaron los primeros oftalmólogos que hubo en la capital del país y que partirían a trabajar al resto de México. Asimismo, este hospital se convirtió en el primer lugar en el que se enseñaría Oftalmología y en la que se entrenarían los oftalmólogos que en 1893 fundaron la Sociedad Oftalmológica Mexicana.

Palabras clave: Hospital. Oftalmología. Oftalmólogos. México. Siglos XIX-XX.

\begin{abstract}
In 1750 was founded in Mexico City an office to attend eye diseases, that together with the service in the Hospital de Pobres gave attention to ocular sickness in Mexico City. Later was founded the Instituto Valdivielso, called some years later Hospital Oftalmológico de Nuestra Señora de la Luz. In this hospital learned the first ophthalmologists who worked in the capital of the country, and later in all Mexico. This hospital was the first institution in Mexico to traine the ophthalmologists who founded the Mexican Ophthalmological Society.
\end{abstract}

Key words: Hospital. Ophthalmology. Ophthalmologists. Mexico. XIX-XX centuries.

Correspondencia:

*Rolando Neri-Vela

E-mail: drnerivela@ hotmail.com CC BY-NC-ND (http://creativecommons.org/licenses/by-nc-nd/4.0/).
Disponible en internet: 06-10-2016 Rev Mex Oftalmol. 2018;92(1):32-38 www.rmo.com.mx
Fecha de aceptación: 31-08-2016 DOI: 10.1016/j.mexoft.2016.08.005 
Un aspecto bien importante que vale la pena señalar en el cuidado de los enfermos de los ojos es que en 1750 , en la ciudad de México, y gracias a la generosidad del conde de Regla Romero de Terreros, en la casa de ejercicios espirituales de los jesuitas, conocida con el nombre de Ara Coeli, se fundó un pequeño establecimiento para padecimientos de la vista, que más tarde se conocería como el consultorio de la señora Fagoaga, y que tras un abandono posterior, fue habilitado por los jesuitas, para que se estableciera posteriormente el hospital de San Andrés ${ }^{1-3}$.

Ricardo Vértiz (Fig. 1), quien ejerció como médico en los hospitales de Jesús y Concepción Béistegui, contribuyó a la medicina mexicana al introducir el método de Lister en las operaciones oculares; en las de catarata usó las curaciones bóricas, aunque con poco éxito, así como las fénicas al milésimo, con cuyo apósito decía que los resultados eran muy felices, ya que no se había complicado ni un solo operado, siendo la regla la cicatrización inmediata. Decía que los enfermos sanaban completamente a los 4 o 5 días, y que hubo casos curados completamente en 2 días y medio; cuando la cicatrización se hacía esperar, no había supuración ni catarro conjuntival, y en las iridectomías usaba las curaciones fénicas, siempre con magníficos resultados ${ }^{4}$.

Ricardo Vértiz, interesado profundamente en las enfermedades de los ojos, realizó una labor extraordinaria para lograr fundar lo que actualmente es el Hospital Oftalmológico de Nuestra Señora de la Luz; durante muchos años dirigió el servicio oftalmológico del Hospital de San Andrés y el 1 de julio de 1884 el presidente de la República lo nombró profesor de Oftalmología en la Escuela Nacional de Medicina, con el sueldo anual de $\$ 1,200.85^{5}$.

Sin embargo, Ricardo Vértiz también tuvo detractores, como lo dice una nota, que casi nunca concurría al Hospital de San Andrés a dar la consulta gratuita para los enfermos de los ojos, como lo tenía anunciado, y que la clínica oftalmológica también se había quedado platicada, no obstante tanto ruido y tantos anuncios en los periódicos, y que era de sentirse, pues el Sr. Vértiz era una persona muy competente ${ }^{6}$. En 1884, en el periódico La Escuela de Medicina, se había anunciado que en el Hospital de San Andrés se había arreglado un departamento especial para servir como clínica oftalmológica, que estaba bien montada, contaba con 8 camas, una sala de espera, otra de operaciones y consulta, y otra para operados, existiendo todos los instrumentos necesarios, y que era asistida por Ricardo Vértiz, a partir de las 9 de la mañana?.

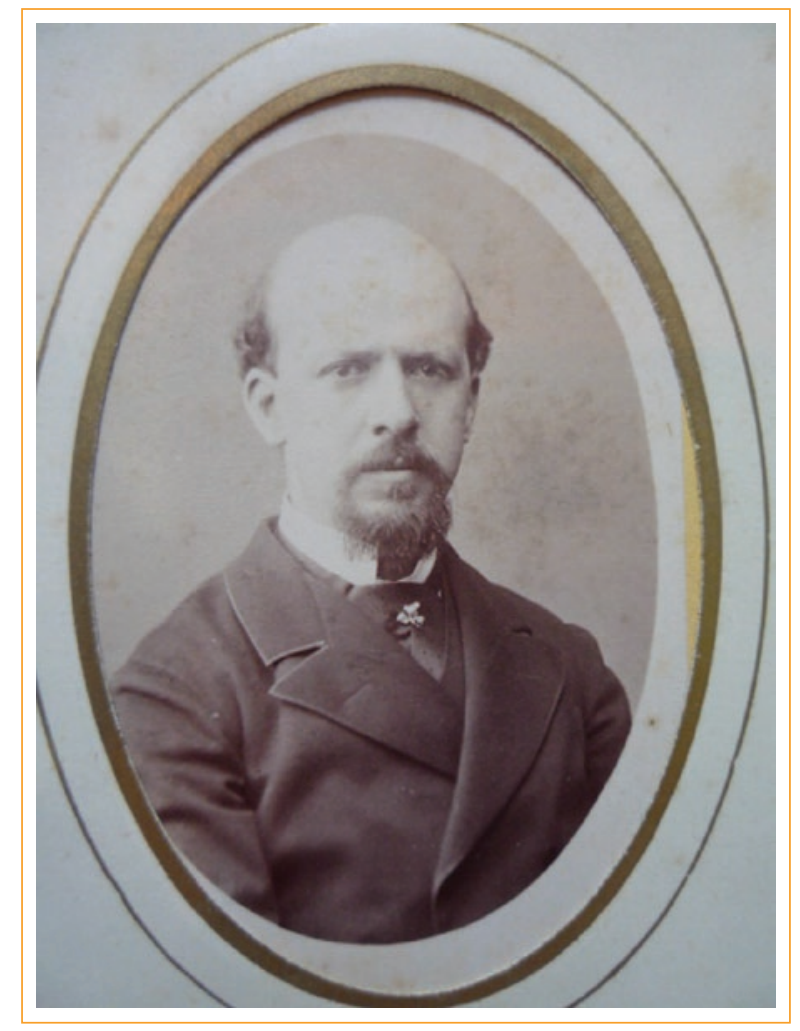

Figura 1. Ricardo Vértiz. Archivo histórico de la Facultad de Medicina.

Daniel M. Vélez (Fig. 2), otro connotado médico, se inscribió en la Escuela de Medicina el 28 de diciembre de 1882, siendo además practicante de la Escuela Práctica Médico Militar. Su tesis versó acerca de «Higiene de la vista»".

De la hoja de servicios de Daniel Vélez que se encuentra en el Archivo Histórico de la Facultad de Medicina de la Universidad Nacional Autónoma de México se desprende lo siguiente: El 6 de marzo de 1895 fue nombrado médico encargado de los trabajos anatómicos en la comisión que debía formar un museo anatomo-patológico en el Hospital de San Andrés, escribiendo como tesis de concurso La asepsia y antisepsia en la cirugía ocular. Este 1895 fue nombrado profesor honorario consultor de Oftalmología. El 10 de abril de 1899 se lo nombró profesor adjunto de Oftalmología teórico-práctica, en la Escuela Nacional de Medicina, y el 23 de agosto de 1901 el presidente de la República lo designó como profesor interino de Clínica Oftalmológica, con el sueldo anual de $\$ 1,200.85$, durante la licencia de un mes que se había concedido al oftalmólogo Agustín Chacón. Para el 21 de abril de 1920, por acuerdo del presidente de la República, se le concedió 




Figura 2. Daniel M. Vélez. Archivo histórico de la Facultad de Medicina.

licencia sin goce de sueldo, a partir del 1 de dicho mes, para separarse del cargo de profesor de Clínica de Oftalmología en la Facultad de Medicina, por el tiempo que desempeñara el de profesor de la misma asignatura en la Facultad de Altos Estudios. El 1 de mayo de 1920 se pidió autorización al director del Hospital General para que dictara la cátedra de Oftalmología de la Facultad de Altos Estudios, en el mismo pabellón que lo hacía el doctor Emilio F. Montaño, profesor de la asignatura en la Facultad. El 2 de enero de 1923 el presidente constitucional de los Estados Unidos Mexicanos lo nombró profesor de Clínica de Oftalmología .

El 31 de agosto de 1925 se le comunicó que, en vista de lo numerosa que había sido la inscripción al segundo trimestre de Oftalmología, se había acordado dividir a los alumnos en 2 grupos, uno de los cuales quedaba a su cargo ${ }^{10}$.
Vélez estudió con dedicación especial los diversos temas de la oftalmología, abordando asuntos como los de la higiene y profilaxis de los ojos de los recién nacidos, la higiene ocular en las escuelas y los cines, los accidentes oculares del trabajo, la educación racional de los ciegos, siendo él quien introdujo en México, para la enseñanza de la lectura a los ciegos, el método de Braille. En 1907 introdujo en México la retinografía ${ }^{11}$.

Manuel Uribe Troncoso nació en la ciudad de Toluca, en donde realizó sus estudios preparatorios, previos a la carrera de medicina, en el Instituto del Estado de México $^{12}$. El 26 de diciembre de 1884 solicitó su ingreso a la Escuela Nacional de Medicina ${ }^{13}$.

En 1899 fue designado médico del Hospital Oftalmológico de Nuestra Señora de la Luz y en 1900 profesor adjunto, por oposición, de la Cátedra de Oftalmología en la Escuela Nacional de Medicina; en 1915 fue profesor titular de Oftalmología clínica en el mismo plantel.

El 11 de diciembre de 1899 el presidente de la sección de Oftalmología de la Asociación Médica Americana le dirigió una carta invitándolo para leer un trabajo sobre «Terapéutica local», bajo el título general de «El uso racional y limitaciones de las medidas terapéuticas necesarias para promover la absorción de los exudados dentro del ojo» en la reunión que dicha asociación celebraría en Atlantic City, del 5 al 8 de junio de $1900^{14}$. Ese año solamente fueron invitadas 2 personas ajenas a la American Medical Association, para presentar sendos trabajos, Juan Santos Fernández y Hernández, de La Habana, y Manuel Uribe, de México ${ }^{15}$. Manuel Uribe Troncoso solicitó al gobierno federal recursos económicos para poder asistir a la reunión, monto que él calculó en 350 pesos oro, ayuda que le fue concedida ${ }^{15}$. A su retorno, Uribe presentó un informe minucioso acerca de la junta, refiriendo que asistieron a la sección de Oftalmología 130 oculistas, tratándose temas tales como la visión entre los trabajadores ferrocarrileros, la ergooftalmología y las operaciones en las cataratas secundarias, y que antes de regresar a México había visitado el Wills Eye Hospital, el New York Eye and Ear Infirmary, el New York Ophthalmic Institute y el Manhattan Eye and Ear Hospital, reflexionando acerca de la higiene visual en las escuelas mexicanas ${ }^{16}$.

Uribe escribió, de 1890 a 1916, 109 trabajos de los 200 que en total publicó; de ellos, 35 muy importantes sobre diversos temas de medicina general e higiene. Los 74 restantes versan sobre oftalmología. José A. Quiroz resaltó que en particular hay que referirse a los relacionados con la filtración del ángulo de la cámara anterior (1905), que dieron lugar a una apasionada polémica con 
el eminente profesor Leber de Heidelberg, quien finalmente convino que Uribe tenía la razón ${ }^{17,18}$.

En 1903, a propuesta de Uribe, se estableció el examen reglamentario de la vista en las escuelas primarias de México ${ }^{19}$.

Uribe publicó, ya en pleno siglo XX un Tratado sobre afecciones internas del ojo y un Atlas oftalmoscópico, del que se hizo una buena traducción española por el Dr. Sanz Astolfi20. Se le debe además a Uribe Troncoso la fundación de la primera revista oftalmológica publicada en español en Hispano-América: los Anales de la Sociedad Mexicana de Oftalmología, publicados en 1898, o sea 3 años antes que los Archivos de Oftalmología Hispano-Americanos fundados por Menacho, de Barcelona, García Calderón, de Madrid, Santos Fernández, de La Habana, y Demicheri, de Montevideo ${ }^{21}$.

Como todo un experto que fue en óptica física y fisiológica, el 14 de mayo de 1919 Emilio Montaño concluyó su trabajo titulado Pequeña contribución al estudio del tratamiento del queratocono, donde explica que este padecimiento afecta a la córnea y provoca miopía, y que se podía remediar ante los inconvenientes de los vidrios combinados y los vidrios de contacto, que eran difíciles de soportar más de una hora, proponiendo que se utilizara la trepanación de Elliot, que permitía tener abatida la tensión intraocular por medio de la comunicación directamente de la cámara anterior del ojo con los linfáticos del corion subconjuntival; este trabajo se publicó al año siguiente en la Gaceta Médica de México ${ }^{22}$.

Emilio Montaño fue oculista del Consultorio Central de la Beneficencia Pública en las calles de Revillagigedo de la ciudad de México.

Enrique Graue Glennie, quien nació en la ciudad de México, se graduó en la Escuela Nacional de Medicina en 1897, después de haber permanecido durante varios años en el Hospital de San Andrés y en el pabellón anexo para enfermos de los ojos, que sería el Instituto Valdivielso, a cargo de Ricardo Vértiz. Además, fue practicante en el Hospital Concepción Béistegui ${ }^{23}$.

Otro afamado oftalmólogo mexicano fue José Ramos, quien nació en San Luis Potosí, especializándose en Europa, y a su regreso a México impartió la cátedra de la especialidad. En París fue jefe de clínica del doctor Galezowski. En 1893 fue parte del grupo de médicos fundadores de la Sociedad Oftalmológica Mexicana. En la Academia Nacional de Medicina llegó a ocupar la presidencia de la institución, en 1896. Falleció el 26 de febrero de 1909.

Ramos fue, probablemente, el primero en el mundo en describir el cisticerco en el ojo ${ }^{24}$.
A fines del siglo XIX y principios del XX, José Ramos tenía a su cargo la cátedra de Patología Interna, en la Escuela Nacional de Medicina, aun siendo su especialidad la oftalmología; sin embargo, sus lecciones fueron famosas por su facilidad de palabra, su dicción elegante y por su elocuencia, unida a una admirable erudición ${ }^{25}$.

La cirugía oftalmológica mexicana de fines del siglo XIX estaba emparejada con la europea, a tal punto que el 8 de noviembre de 1899 el doctor Lorenzo Chávez presentó ante la Academia Nacional de Medicina una enferma que había operado de miopía fuerte por medio de la supresión del cristalino transparente, en los dos ojos, afirmando su creencia de que era esa «la primera operación de este género, que, de una manera intencional y pensada», se practicaba en México ${ }^{26}$. Lorenzo Chávez fue director del Hospital Oftalmológico de Nuestra Señora de la Luz de 1898 a 1911.

De familia humilde, en un pequeño poblado de los Altos de Jalisco, nació José de Jesús González. Inició sus estudios de medicina en la ciudad de México, asistiendo con regularidad al Servicio de Oftalmología del Hospital de San Andrés, lo que le permitió conocer los secretos de la especialidad.

En 1892 Jesús Chico, secretario y director interino de la Escuela del Estado de Guanajuato, certificó que en ese año había obtenido en el examen de Anatomía descriptiva 3 votos de perfectamente bien, así como en Histología, y en Farmacia elemental 2 votos de perfectamente bien y uno de muy bien ${ }^{27}$.

En 1894 José de Jesús González escribió una carta al director de la Escuela Nacional de Medicina, misma que transcribo para mostrar la conducta que tenía, respetando la ortografía correspondiente:

C. Director de la Escuela Nacional de Medicina de México

José de Jesús González, vecino de San Francisco del Rincón, Estado de Guanajuato, ante usted, salvas las protestas más oportunas y como mejor proceda, comparezco y expongo:

El año próximo anterior tuve la honra de acreditar ante la Escuela de que usted es digno Director, todos mis estudios preparatorios y de primer año de medicina, cursado en la escuela respectiva de Guanajuato, á fin de que se me concediera exámen de las materias correspondientes á segundo año, que cursé en la escuela de medicina de esa capital, como alumno supernumerario, en las cuales materias fui unanimemente aprobado, como es de verse en las actas respectivas de seis y nueve de noviembre próximo pasado. 
Abrigando, pues, ahora un deseo vehemente de cursar tercer año; y no pudiendo por atenciones graves de familia presentarme luego á esa Escuela á sacar mi matrícula, me permito el honor de suplicar á usted muy atentamente se digne concederme la gracia de que se me matricule en dicho tercer año.

Juzgo arreglada mi solicitud y por tanto a usted respetuosamente suplico se digne proveer de conformidad con lo que recibiré gracia que impetro; permitiéndome además suplicar á usted se sirva tener á bien disponer que se reserve mi matrícula con Secretaria para que se me entregue á la hora que me presente á pedirla.

También ruego a usted se sirva admitirme con la estampilla de que he hecho uso por ser notoria mi pobreza.

\section{Protesto no obrar de malicia.}

San Francisco del Rincón, trece de enero de mil ochocientos noventa y cuatro.

José de Jesús González.

La solicitud fue aprobada con fecha 30 de enero de $1894^{28}$.

El 26 de marzo de 1897, González solicitaba su examen general de Medicina, Cirugía y Obstetricia, mismo que sustentó el 31 de mayo de 1897, a las 6 de la tarde. Los sinodales propietarios fueron Joaquín Vértiz, A. Ruiz Erdozain, M. Zúñiga, Ángel Gaviño y Domingo Orvañanos, y el suplente, Secundino Sosa ${ }^{29}$.

José de Jesús González acudió durante 3 años a la clínica oftalmológica del doctor José Ramos, en el hospital de San Andrés, acompañando además a Lorenzo Chávez como practicante numerario en el Hospital Béistegui en sus operaciones oftalmológicas.

González regresó a León, instalando su consultorio en la calle Pacheco, que en la actualidad se llama 5 de Mayo. Se dedicó a la medicina general y más tarde a la oftalmología.

El 12 de noviembre de 1907 solicitó el examen a la Escuela Nacional de Medicina para obtener el título de especialista en Oftalmología, anexando una lista de sus trabajos originales sobre oftalmología publicados, y una carta del Dr. José Ramos, haciendo constar la dedicación y aprovechamiento de José de Jesús González ${ }^{30}$. Su sapiencia era tal que hablaba latín, inglés, catalán, portugués, italiano y francés ${ }^{31}$.

Ignacio Valdivieso y Vidal de Lorca, que vivió varios años en París, en donde murió el 17 de mayo de 1861, en testamento otorgado en agosto de 1857 y depositado en la legación de México, instituyó un legado a favor de los pobres de la ciudad de México, por la cantidad de 7,900 francos anuales, que había de quedar constituido en un título, inalienable, de la renta francesa del $3 \%$, encargando como albacea al señor Juan Francisco Allsopp, inglés residente en la ciudad de México, el que hiciera lo necesario para la aplicación del legado.

El Sr. Allsopp, después de los largos trámites legales de la testamentaría decidió, como albacea y ejecutor testamentario, dedicar esa renta a la atención de los pobres, enfermos de los ojos. Para ello, y aprovechando que había sido derribada la iglesia anexa al Hospital de San Andrés, en la planta baja de la fachada oriental de dicho hospital, construyó un departamento autónomo, destinado a la atención de afecciones oculares, que se denominó Instituto Valdivieso, contando con una sala para 10 enfermos del sexo masculino y que fueran pobres, y 2 departamentos, uno de consulta y el otro para el examen de los pacientes, que servía también de curaciones y para operaciones. La dirección médica fue encomendada a Agustín Andrade, inaugurándose el 15 de mayo de 1876. Las mujeres, por su lado, eran atendidas en el Hospital de Jesús ${ }^{32}$.

Juan Francisco Allsopp quedó nombrado patrono de la institución, según resolución del Juzgado $2 .^{\circ}$ de lo Civil de la ciudad de México, con fecha 21 de noviembre de 1889. Muerto Allsopp, el mismo juzgado, por auto de fecha 21 de noviembre de 1891, nombró al licenciado Rafael Dondé, ilustre jurisconsulto del Foro Mexicano, para sustituirlo como albacea y patrono de la Institución Valdivieso ${ }^{15}$.

Mientras tanto, y bajo la dirección de Andrade, el nosocomio fue creciendo en fama y el número de pacientes aumentó. En el Instituto, Manuel Carmona y Valle dictó las primeras clases de Oftalmología, en donde se formaron, entre otros muchos, Agustín Chacón, Ricardo Vértiz, José Ramos y Federico Ábrego, José María Gama, Emilio Montaño, Manuel Uribe Troncoso, Lorenzo Chávez, Fernando López y Sánchez Román, Ignacio del Valle, Enrique Graue Glennie, Daniel Vélez y José de Jesús González ${ }^{15}$.

El 3 de enero de 1889 Manuel Carmona y Valle, en su carácter de director de la Escuela Nacional de Medicina, solicitó al encargado del Hospital Valdivielso que se permitiera que allí se dictara la Clínica oftalmológica ${ }^{33}$. Al pedido de Carmona y Valle, Juan Francisco Allsopp contestó afirmativamente, con la condición de que la Escuela no se inmiscuyera en la organización y la administración del hospital, nombrando a Joaquín Vértiz para que redactara el reglamento respectivo ${ }^{15}$. 
A partir de este momento, la enseñanza de la oftalmología en México se hizo más formal aun.

Además, el filántropo mexicano Francisco Somera, quien había muerto en la ciudad de México en mayo de 1889, había hecho su testamento el 14 de marzo de 1888 , y en él nombró por herederos de su cuantiosa fortuna a las siguientes instituciones benéficas establecidas en la capital de la República: Hospital de Jesús Nazareno, Sociedad de Beneficencia Española, Asociación de Beneficencia Francesa, Suiza y Belga, Hospital Americano e Institución Oftalmológica Valdivielso, en la proporción del número de camas que tuviera cada una de ellas a la muerte del testador; para esa fecha la institución tenía 10 camas $^{34}$.

El correr inclemente de los días se hizo presente, y con ello la necesidad de contar con un local más amplio; al no poder extenderse más en lo que era el Hospital de San Andrés, Vértiz pensó en lo conveniente que era tener un nosocomio, poniendo a colaboración a sus muchas amistades y relaciones médicas, logrando obtener la suma de $\$ 10,211.00$, con lo que adquirió un terreno de 1,817 metros cuadrados en lo que entonces era el límite de la ciudad, en la calle de La Paz, hoy Ezequiel Montes, empezando la construcción del hospital, proyectado por el arquitecto Manuel Calderón ${ }^{35}$.

En 1894 la muerte sorprendió al doctor Ricardo Vértiz, pero por encargo expreso suyo, su ejecutor testamentario, Félix Cuevas, continuó con la construcción de la obra, y no solo eso, sino que habiéndose agotado los fondos recabados por el doctor Vértiz, el mismo Félix Cuevas dio de su bolsa las cantidades faltantes para terminar la parte principal del edificio, que alcanzaron más de $\$ 17,500.00$, poniendo como condición que no debía decirse quién había dado ese dinero, hasta después de su muerte ${ }^{15}$.

Mientras, Joaquín Vértiz, hermano de Ricardo Vértiz, lo suplió al frente del Instituto. Terminada la parte principal de la construcción, Félix Cuevas, en su cargo de albacea testamentario de Ricardo Vértiz, y de acuerdo con Rafael Dondé, patrono del Instituto Valdivieso desde 1891, se pusieron de acuerdo para dar forma legal a la fusión y continuidad de la institución, con el nuevo edificio del hospital creado, y habiendo obtenido de la autoridad correspondiente el permiso necesario, fueron trasladados al nuevo hospital los enfermos que sostenía la Institución Valdivieso, abriendo sus puertas al público el 27 de octubre de 1898, bajo la dirección de Lorenzo Chávez, quien tuvo como ayudantes a Enrique Graue Glennie e Ignacio del Valle; como patrono único continuó Rafael Dondé ${ }^{15}$.
El Instituto Valdivieso cambió su nombre a Hospital de Nuestra Señora de la Luz. Institución de Oftalmología Valdivieso a sugerencia de una dama que obsequió un cuadro de la Virgen Santísima de la Luz, de tamaño natural, pintura original de Miguel Cabrera, que se conserva y venera aún en la capilla del hospital.

A principios del siglo XX, la señora Dolores Quintanilla de Orvañanos construyó a sus expensas el resto de la obra proyectada, consistente en una sala de operaciones, 2 salas para enfermos pobres, 2 para enfermos de alguna mejor posición económica y otras dependencias más. Quedó así completo el edificio del nosocomio, con capacidad para 46 enfermos asilados, salas de consulta, de operaciones y de curaciones ${ }^{15}$.

Ya establecida la institución, sus fundadores y patronos, Rafael Dondé, Félix Cuevas, Dolores Quintanilla de Orvañanos y Mariano Prado (yerno de Vértiz), quisieron que se perpetuara la fundación, para lo que la sujetaron a las prescripciones de la Ley de Beneficencia Privada del 7 de noviembre de 1899. Para este efecto, se levantó ante el notario Agustín Pérez de Lara el acta constitutiva del Hospital de Nuestra Señora de la Luz, con fecha 19 de junio de 1902. Ha gozado de personalidad jurídica desde que se extendió el decreto del Poder Ejecutivo de fecha 31 de diciembre de 1904.

En 1888 se iniciaron las clases de perfeccionamiento médico en la Escuela Nacional de Medicina; Ricardo Vértiz impartió Oftalmología teórica y práctica, usando como texto el libro de Mayer ${ }^{36}$. En 1894 impartió la asignatura José Ramos, los martes, jueves y sábados, de 9:00 a 10:30 horas, y los textos eran los de Galezowski y de Dagennet ${ }^{15}$.

Así, esta institución se convirtió en la semilla que daría como frutos la formación de otros hospitales y servicios de Oftalmología en todo el país.

\section{Financiamiento}

Los autores no recibieron patrocinio para llevar a cabo este artículo.

\section{Conflicto de intereses}

Los autores declaran no tener ningún conflicto de intereses.

\section{Bibliografía}

\footnotetext{
1. Ruz M. Chiapas colonial: dos esbozos documentales. México: Universidad Nacional Autónoma de México; 1989
} 
2. Flores, Troncoso F. Historia de la medicina en México, tomo II. México: Instituto Mexicano del Seguro Social; 1982.

3. Santiago F. Los hospitales de México y la caridad de Don Benito. Figuras y episodios de la historia de México, n ${ }^{\circ}$ 67. México: Editorial Jus; 1959.

4. Flores, Troncoso F. Historia de la medicina en México. Tomo III. México: Instituto Mexicano del Seguro Social; 1982.

5. AHFM, UNAM, FEMYA, leg. 43, exp. 5, f. 8 .

6. La Escuela de Medicina, Tomo VI, n. ${ }^{\circ} 21$, mayo 1 de 1885, p. 288

7. La Escuela de Medicina, Tomo VI, n. ${ }^{\circ} 7$, octubre 1 de 1884 , p. 98.

8. La Escuela de Medicina, Tomo X, n. ${ }^{\circ}$ 10, julio 15 de 1889, p. 205.

9. AHFM, UNAM, FEMYA, leg. 56 , exp. 12

10. Ídem.

11. Vélez D, Martínez F. Apuntes para la historia de la oftalmología en México. Anales de la Sociedad Mexicana de Oftalmología. 1940:6.

12. AHFM, leg. 38 , núm. 11, f. 1 .

13. Ibídem, f. 2.

14. CESU, Fondo Escuela Nacional de Medicina, caja 29, exp. 61, fs. 183-191.

15. Idem.

16. Ibídem, fs. $193-207$

17. Quiroz J. Recuerdo del doctor Manuel Uribe y Troncoso. Anales de la Sociedad Mexicana de Oftalmología. 1959;1:6-7.

18. Martínez F. Notas históricas de oftalmología en el cuatricentenario de la Universidad de México. En: IV Centenario de la Universidad de México (1551-1951). Memoria del Congreso Científico Mexicano. VIII. Ciencias Médicas. México: Universidad Nacional Autónoma de México; 1953. p. 145-51.

19. Gortari E. La ciencia en la historia de México. 3. ${ }^{a}$ ed. México: Editorial Grijalbo; 1980.
20. Márquez M. El Prof. D. Manuel Uribe Troncoso. Homenaje. Anales de la Sociedad Mexicana de Oftalmología. 1959;1:10.

21. Ibídem, p. 11

22. Sánchez G. Efemérides de mayo. Gaceta de la Facultad de Medicina. 2001:14.

23. AHFM, Fondo Escuela de Medicina y alumnos, leg. 63, exp. 35, f. 4.

24. Alcocer A, Alonso AF. Biobibliografía. Archivos de Historia Potosina. 1976;2:126-7.

25. Ocaranza F. Historia de la medicina en México. México: Consejo Nacional para la Cultura y las Artes; 1995.

26. Valle RH. La cirugía mexicana del siglo XIX. México: Editorial tipográfica Sag; 1942.

27. AHFM, Fondo Escuela de Medicina y Alumnos, leg. 65, núm. 44, f. 1

28. Ibídem, f. 8 y 8 v.

29. Ibídem, f. 12

30. Ibídem, f. 13 .

31. Gómez E. Historia de la medicina de León. México: Editorial PAC S.A. de C. V.; 2000.

32. Graue E. La oftalmología en México en los últimos cien años. Boletín del Hospital Oftalmológico de Nuestra Señora de la Luz. 2007;214: 17-21.

33. CESU, Fondo Escuela Nacional de Medicina, caja 34, exp. 2, fs. 5-9.

34. Olivera FJ. El Hospital de Nuestra Señora de la Luz. Divulgación Histórica. 1943;IV:208-13

35. Graue, op. cit.

36. Ruiz L. Apuntes históricos de la Escuela Nacional de Medicina. México: Universidad Nacional Autónoma de México. Facultad de Medicina; 1963. 